\title{
Zonificación de áreas potenciales para el desarrollo de sistemas silvopastoriles en la microcuenca Lluchca,
} Chachapoyas, Amazonas

\section{Zoning of potential areas for the development of silvopastoril systems in the microbasin Lluchca, Chachapoyas, Amazonas}

\author{
Jhovana Sopla Mas ${ }^{1}$, Nilton Beltrán Rojas Briceño ${ }^{1}$, Gerson Meza Mori ${ }^{1}$, Rolando Salas López ${ }^{1 *}$
}

\section{RESUMEN}

El avance de las prácticas ganaderas a nivel mundial está creciendo exponencialmente. Consecuentemente a esto tenemos las pérdidas de bosques, quedando cada vez más desérticas los suelos, afectando la micro fauna y flora presente en la misma. El objetivo principal fue determinar áreas potenciales para el desarrollo de sistemas silvopastoriles (SSP) mediante la utilización de Sistemas de Información Geográfica en la microcuenca Lluchca, provincia de Chachapoyas. Para ello se definieron cuatro tipos de aptitud: muy bueno, bueno, regular y malo. Para la delimitación de la microcuenca Lluchca se emplearon herramientas SIG (Sistemas de Información Geográfica), que facilitaron la manipulación y superposición de capas temáticas de tipo de suelo, pendiente, capacidad de uso mayor, zona de vida, red vial, red hídrica, precipitación y elevación. A estas capas se le aplicó un análisis multicriterio Proceso analítico Jerárquico (AHP), donde se encontraron zonas con aptitud buena en 146 hectáreas, regular en 122 hectáreas y mala en 102 hectáreas, sin embargo no se encontraron zonas con aptitud óptima o muy buena.

Palabras claves: AHP, zonificación, SSP, SIG, microcuenca.

\section{ABSTRACT}

The advance of livestock practices worldwide is growing exponentially, consequently we have the loss of forests, the soils become increasingly desert, affecting the micro fauna and flora present in it, the main objective was to determine potential areas for the development of silvopastoral systems (SSP) through the use of Geographic Information Systems in the Lluchca microbasin, Chachapoyas province. For this, four types of aptitude were defined: very good, good, regular and bad. For the delimitation of the Lluchca micro-basin, GIS (Geographical Information Systems) tools were used, which facilitated the manipulation and overlapping of thematic layers of soil type, slope, capacity of major use, life zone, road network, water network, precipitation and elevation, a multicriterion analysis was applied to these layers - Hierarchical Analytical Process (AHP), where areas with good aptitude were found 146 hectares, regular 122 hectares and bad 102 hectares, however there were no zones with optimal aptitude or very good.
\end{abstract}

Keywords: AHP, zoning, SSP, GIS, microbasin. 


\section{INTRODUCCIÓN}

La degradación de los suelos es un problema que es causado por deforestación, sobrepastoreo, y monocultivo, entre otros (Mazo et al., 2016). Sus consecuencias son la pérdida de nutrientes, erosión, degradación física y aumento de escorrentía (Abdelrahman, et. al., 2016). Así surgen los sistemas silvopastoriles (SSP) como una alternativa de desarrollo sostenible. Los SSP generalmente se diseñan sobre bosques secundarios degradados (Silberman et al., 2015), y a su vez permiten un mejor aprovechamiento de los nutrientes del suelo aumentando la disponibilidad del pasto (Alonso, 2011).

La ganadería es una actividad que aporta un tercio de las proteínas humanas (Sakadevan y Nguyen, 2017), y la misma densidad de ganado conlleva al sobrepastoreo (Martínez-Valderrama et al., 2017), esta actividad incrementa la desertificación (Bo et al.y, 2013). La reducción de gramíneas reduce la cantidad de materia orgánica (Ibañez et al., 2007), y reduce significativamente la retención de agua (Li et al., 2016). Es así que existe la necesidad para luchar contra los problemas de desertificación (Martínez-Valderrama et al., 2017).

Por lo que el deterioro de la calidad del suelo necesita prácticas adecuadas de conservación (Abdelrahman et al., 2016) siendo una excelente alternativa los sistemas silvopastoriles con importantes efectos en la fertilidad del suelo (Silberman et. al., 2015). Por otra parte la introducción de leguminosas genera fertilidad y calidad de forrajes en oferta para los animales. Se estima que el uso de leguminosas como abono verde puede contribuir con $30 \mathrm{a} 80 \mathrm{~kg}$ /ha por año de nitrógeno (López y García, 2015).

La zonificación para determinar la idoneidad del suelo y reducir la degradación de la tierra (Angelo et, al., 2000), con el resultado de superposición de mapas usando Sistemas de Información Geográfica (SIG) cada vez son más sólidos (Boccoet al., 2017), permitiendo evaluar fácilmente factores determinantes en la idoneidad de la tierra para el desarrollo de agroforestería (Laray et al., 2017), La recuperación de tierras es altamente efectiva cuando se implementan sistemas agroforestales (Palma-López et al., 2007). Además la tecnología SIG ofrece potencial de evaluación económica, rápida y repetida del suelo a diversas escalas (Obade y Lal, 2013) y el monitoreo de tierras degradadas.

\section{MATERIAL Y MÉTODOS}

\section{Localización del área de estudio}

El estudio se realizó en la microcuenca Lluchca, provincia Chachapoyas, Departamento Amazonas. Ubicada entre las coordenadas 6 $6^{\circ} 14.39^{\prime \prime}$ y $6^{\circ} 4^{\prime} 5.00^{\prime \prime}$ de

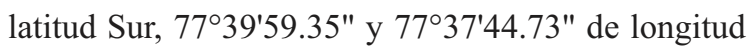
Oeste, zona 18 (Figura 1), en esta microcuenca se tiene gran potencial de prácticas ganaderas, siendo esta la principal actividad del crecimiento económico del distrito Olleros. Geográficamente, el área de estudio se encuentra ubicado en la región de sierra ceja de selva de las estribaciones bajas de la Cordillera Oriental de los Andes. Se encuentra comprendida aproximadamente entre los 2785 y 3395 msnm y, comprende una superficie de 391,70199 ha.

\section{Metodología}

Para determinar la zonificación de áreas potenciales para el desarrollo de SSP en la microcuenca Lluchca, se siguió la metodología de Saaty (2008), cuya parte esencial consistió en determinar el nivel de importancia (peso), de cada variable y estandarizar sus valores en términos de aptitud para el desarrollo de SSP. Las variables intervenidas son: capacidad de uso mayor, tipo de suelo, zonas de vida, uso actual de suelo, disponibilidad hídrica (ríos), pendiente, precipitación, uso actual de suelo y accesibilidad (vías). Para cada variable se generaron capas digitales a una escala de 1:10000 que se integraron y analizaron en ArcGis 10.4.1.

La información de precipitación se obtuvo interpolando cinco estaciones: Olleros, Agua Dulce, Chachapoyas, Cocachimba y Huambo, con datos de 5 años de antigüedad, las cuales fueron procesadas en el software Excel e interpolados en ArcGis 10.4.1 para la generación de capas de isoyeta.

Para los datos de suelo, se realizaron cinco calicatas, 


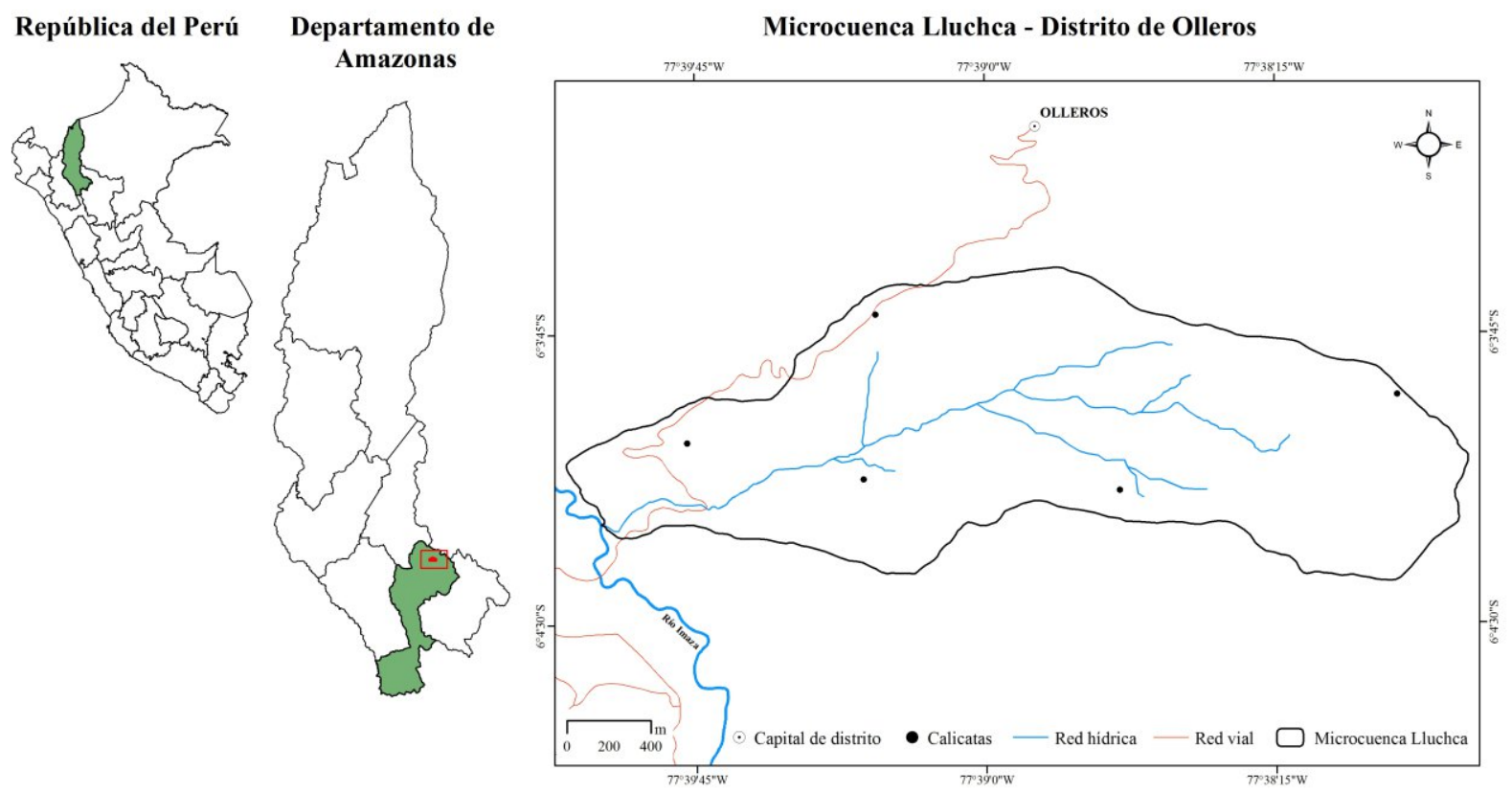

Tabla 1. Ubicación geográfica del área de estudio en la microcuenca del río Lluchca, provincia de Chachapoyas (Departamento de Luya).

ubicadas en cada tipo de fisiografía presente en la microcuenca (Tabla 1), de los cuales se obtuvo información de profundidad, $\mathrm{pH}$, conductividad eléctrica, $\mathrm{P}, \mathrm{K}, \mathrm{C}, \mathrm{MO}, \mathrm{N}$, cationes cambiables, CIC y clase textural.

Tabla 1. Coordenadas de las calicatas tomadas en campo

\begin{tabular}{cccccc}
\hline ID & $\mathbf{X}$ & $\mathbf{Y}$ & Altitud & Banda & Zona \\
\hline 1 & 208635 & 9328886 & 3239 & 18 & $\mathrm{M}$ \\
2 & 207311 & 9328426 & 3056 & 18 & $\mathrm{M}$ \\
3 & 206088 & 9328476 & 2931 & 18 & $\mathrm{M}$ \\
4 & 205244 & 9328646 & 2889 & 18 & $\mathrm{M}$ \\
5 & 206143 & 9329262 & 2950 & 18 & $\mathrm{M}$ \\
\hline
\end{tabular}

Para establecer los puntos de las calicatas se interpolaron capas de pendiente, curvas de nivel, geología, modelo de elevación digital (DEM), analizadas en ArcGis v. 10.4.1. Las muestras se enviaron al Laboratorio de Investigación en Suelos y Aguas del Instituto de Investigación para el Desarrollo Sustentable de Ceja de Selva (INDES-CES), de la Universidad Nacional Toribio Rodríguez de Mendoza de Amazonas (UNTRM-A), para finalmente obtener la capa de tipo de suelo con ayuda del software ArcGis v. 10.4.1.

Para la capa uso actual de suelo se siguió la metodología de IDEAM, IGAC y CORMAGDALENA, 2008. Utilizando con base una imagen Sentienel 2A, para la cual se tomaron 192 aéreas de entrenamiento distribuidas aleatoriamente en toda la cuenca.

Modelado para zonificación de áreas óptimas para el desarrollo de SSP mediante análisis multicriterio Para la jerarquización de variables se elaboró la matriz de comparación por pares basada en la escala de Saaty. Una vez que se definieron los pesos se elaboró el modelo para zonificación de áreas potenciales mediante una combinación lineal ponderada (Malczewski, 1999):

$$
p=\sum_{i=1}^{n} w_{i} x_{i}
$$

Donde:

$\mathrm{P}=$ Aptitud de áreas para el desarrollo de SSP

$\mathrm{W}_{\mathrm{i}}=$ peso del criterio $\mathrm{i}$

$\mathrm{X}_{\mathrm{i}}=$ valor estandarizado del criterio i para cada pixel

Los valores obtenidos se clasificaron en 4 clases de aptitud: muy buena, buena, regular y mala

\section{RESULTADOS}

Se obtuvo el mapa de cada una de las variables necesarias para la zonificación de áreas para el 
desarrollo de SSP. Las variables utilizadas con sus respectivos pesos se muestran en la Tabla 2, donde las variables con mayor peso son la capacidad de uso mayor, tipo de suelo y zona de vida.

Tabla 2. Ponderaciones de los pesos de los factores

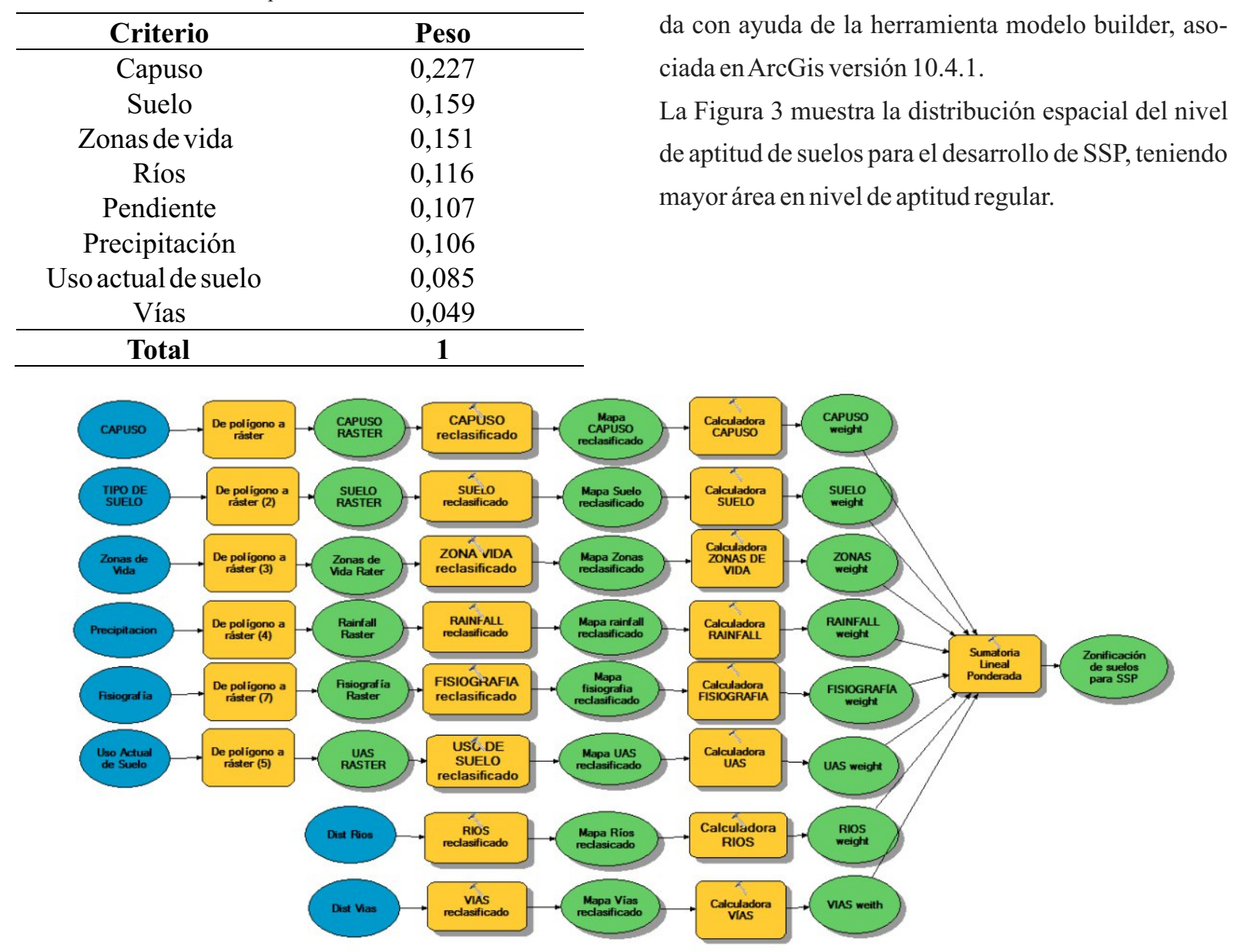

Figura 2. Modelo de idoneidad de tierras para el desarrollo de SSP.

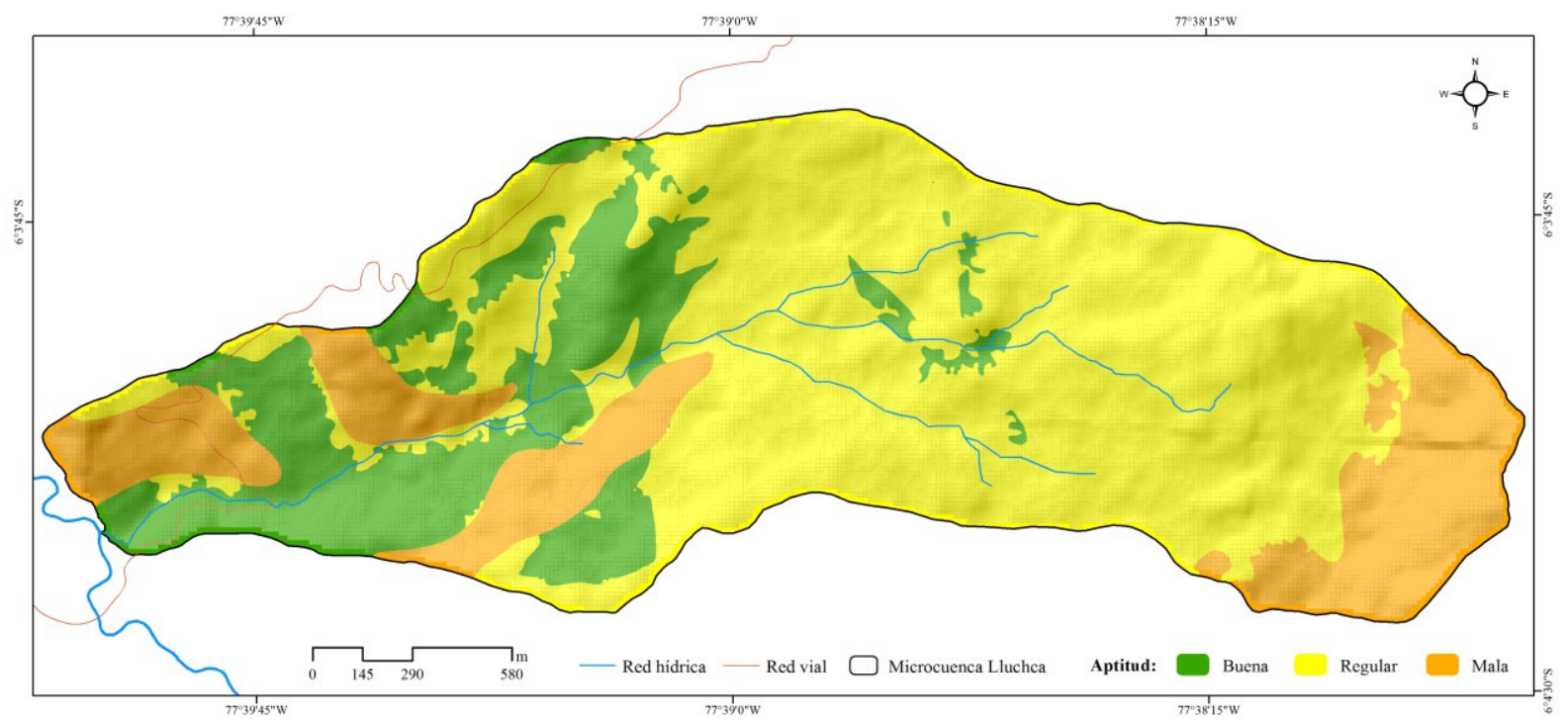

Figura 3. Mapa de Zonificación y distribución de tierras para desarrollo de SSP en la microcuenca Lluchca.
La Figura 2 muestra el modelo para encontrar el mapa de zonificación. Se tuvo que realizar una multiplicación de cada uno de los criterios por sus pesos obtenidos en la matriz de comparación por pares. Seguidamente la sumatoria lineal ponderada, realizada con ayuda de la herramienta modelo builder, asode aptitud de suelos para el desarrollo de SSP, teniendo mayor área en nivel de aptitud regular. 


\section{IV.DISCUSIÓN}

En el analisis de la matriz de comparación por pares, se indica que el peso resultante mayor es la capacidad de uso mayor, seguido de la capa suelo. Demostrando que la calidad de los suelos es un factor muy importante en zonificación de áreas óptimas para el desarrollo de SSP (Sharma et al., 2014). Los siguientes pesos son las capas zonas de vida, ríos, pendiente, precipitación uso actual de suelo y vías.

Los pesos elevados adquiridos para la capacidad de uso mayor y la pendiente según Widiadmaka (2016) tienen efectos de conservación de suelos,. Las áreas de gran altitud por lo general tienen pendientes pronunciadas, por lo tanto se requiere un manejo integrado para potenciar su capacidad productiva en beneficio del hombre, y lograr el desarrollo sostenible (Sánchez et al., 2011).

Los resultados de zonificación se presentan en la Tabla 2, donde se muestra el mapa de zonificación y distribución de tierras para desarrollo de SSP en la microcuenca Lluchca. Sin embargo no se cuenta con áreas ideales, es decir no existen áreas con idoneidad total de acuerdo a las variables aplicadas conforme a Saaty (2008). Teniendo suelos buenos en la parte baja de la cuenca, y siendo las partes altas por déficit en propiedades físicas y químicas del suelo, pendiente muy pronunciada, entre otros factores los que tienen una zonificación de mala calidad para el desarrollo de SSP tal como lo indica (Widiadmaka, 2016). La clase dominante es la que presenta aptitud regular, estos tienen uso actual de suelo con pastos, siendo muy adecuados para convertirlos en SSP para lograr tener suelos con cobertura arbórea para mayor infiltración, menor escorrentía y menores suelos erosionados (Sarasty et al., 2016) y los suelos con un peso bajo o malas son aquellas áreas destinadas principalmente a conservación.

Tabla 3. Distribución de Área de aptitud en la microcuenca Lluchca

\begin{tabular}{cccc}
\hline FID & GRIDCODE & Área & Aptitudes \\
\hline 0 & 1 & 87,68 & Malo \\
1 & 2 & 239,07 & Regular \\
2 & 3 & 64,95 & Bueno \\
\hline
\end{tabular}

Con este estudio se comparte la misma opinión de Delgado et al. (2010) que dice que la evaluación multicriterio implementada en un ambiente SIG, combinada con métodos como AHP, es adecuada para evaluar aptitud de tierras integrando diversos criterios. El proceso analítico jerárquico resulta eficaz para definir jerarquías de los diferentes criterios (Vargas y Ponce de León, 2008) aplicados en un modelo de idoneidad.

El proceso analítico jerárquico se basa en factores y restricciones (Gómez y Barredo, 2005), sin embargo dentro de las restricciones o limitantes, para el presente estudio se tomó: área urbana, explotación de recursos naturales y áreas naturales protegidas. Por otra parte, solo queda en encontrar la idoneidad de tierras con los factores y que en este caso, son cada una de las variables. Los resultados de la evaluación de aptitudes pueden confrontarse con el uso adecuado del suelo (Parrado, 2004)

El uso adecuado del suelo favorece la conservación de las microcuencas, las propuesta de zonificación son básicamente cualitativas, las cuales ayudan a afrontar problemas y posibilidades de actividades agropecuarias (Mora y Ramírez, 2013). Sin embargo el desarrollo de actividades agropecuarias en las zonas ribereñas hace vulnerable a la contaminación de una cuenca o microcuenca (Domínguez, 2008). La zonificación ambiental contribuye al desarrollo sostenible de las micro cuencas (Mora y Ramirez, 2013), y precisamente eso es lo que se busca con la presente investigación. Se comparte la opinión de Delgado et. al., (2007) que indica que los criterios de evaluación en la metodología AHP permiten escoger áreas que, aunque no reúnen la totalidad de las demandas de un factor, o porque éste sea poco relevante de acuerdo con su valor de ponderación, no impactan de forma significativa en la determinación de la aptitud total. En cuanto a la superficie de aptitud por los estrictos criterios establecidos no se encuentran zonas con aptitud ideal.

Los estudios de zonificación resultan adecuados para tener un mayor aprovechamiento de los recursos, buscando la conservación y restauración de los suelos (Cotler et al., 2015). Además la idea de la integralidad 
ecosistémica con SSP, es ideal para simular los efectos de las acciones y políticas ambientales en diferentes escenarios (Coral, 2016). Mejorando la presentación panorámica y conservación de los suelos, con el fin de aumentar la cobertura boscosa y proteger el recurso hídrico sin excluir las actividades socioeconómicas de la zona (Mora y Ramírez, 2013).

\section{CONCLUSIONES}

Los factores intervenidos en el estudio de zonificación de áreas con aptitud fueron: la capacidad de uso mayor, el tipo de suelo, las zonas de vida, disponibilidad hídrica, pendiente, precipitación, uso actual de suelo y vías de acceso.

El uso de análisis multicriterio AHP integrado a un SIG permitió tener resultados de zonificación integrando diferentes variables, las cuales toman mayor peso una frente a la otra, de acuerdo a la escala de Saaty, permitiendo jerarquizar u ordenar las variables por nivel de importancia, siendo la capacidad de uso mayor, el tipo de suelo y la zona de vida, con pesos 0,$227 ; 0,199$ y 0,151 , respectivamente los que toman los valores de mayor importancia.

El análisis de idoneidad para el desarrollo de SSP en la microcuenca Lluchca se basó en la interpolación de ocho criterios, clasificados en suelos buenos, regulares y malos, encontrando la mayor extensión en áreas con aptitud regular 239,07h a para el desarrollo de SSP.

El resultado de distribución espacial para el desarrollo de SSP muestra los lugares con las condiciones más óptimas para el desarrollo de este tipo de actividad, ayudando grandemente a un crecimiento y desarrollo ordenado en cuanto a la ocupación del suelo, favoreciendo la así conservación del mismo.

\section{REFERENCIAS BIBLIOGRÁFICAS}

Abdelrahman, M. A. E., A. Natarajan, y C. A. Srinivasamurthy. 2016. "Estimating Soil Fertility Status in Physically Degraded Land Using Gis and Remote Sensing Techniques in Chamarajanagar District, Karnataka, India.”
The Egyptian Journal of Remote Sensing and Space Science 19 (1): 95-108. doi: 10.1016/j.ejrs.2015.12.002

Alonso, J. 2011. "Los Sistemas Silvopastoriles y su Contribución al Medio Ambiente." Revista Cubana de Ciencia Agrícola 45 (2): 107-115.

Angelo, M., G. Enne, S. Madrau, y L. Percich. 2000. "Mitigating Land Degradation in Mediterranean Agro-Silvo-Pastoral Systems : a GisBased Approach.” CATENA 40 (1): 37-49.

Bo, T., L. Fu, y X. Zheng. 2013. "Modeling the Impact of Overgrazing On Evolution Process of Grassland Desertification. Aeolian Research 9: 183-189.

Bocco, G., A. Velázquez, y K. Gajewski. 2017. “On the Relationship between Landforms and Land Use in Tropical Dry Developing Countries. A GIS and Multivariate Statistical Approach." Investigaciones Geográficas: Boletín del Instituto de Geografía 2017 (93), 3-19. doi:10.14350/rig.56438

Coral, A. C. 2016. "Zonificación En Cuencas Hidrográficas Para La Implementación De Políticas De Incentivos A La Conservación Y Restauración De Ecosistemas. Caso Cuenca Hidrográfica Del Río Buena Vista, Ecuador." Revista Entorno Geográfico (12): 50-68.

Cotler, H., S. Cram, S. Martínez, y V. Bunge. 2015. "Evaluación De Prácticas De Conservación De Suelos Forestales En México: Caso De Las Zanjas Trinchera." Investigaciones Geográfi$\operatorname{cas}$ (88): 6-18.

Delgado, C. E., J. R. Valdez, A. M. Fierros González, H. M. Posadas, y A. Gómez. 2010. Aptitud De Áreas Para Plantaciones De Eucalipto En Oaxaca Y Veracruz: Proceso De Análisis Jerarquizado Vs. Álgebra Booleana. Revista Mexicana De Ciencias Forestales 1 (1): 123133.

Domínguez, S. 2008. “Zonificación Ambiental Para El Ordenamiento Territorial De La Subcuenca 
Bimunicipal Del Rio Aguas Calientes, Nicaragua." Recursos Naturales y Ambiente (55): 64-73

Gómez, M., y J. I. Barredo. 2005. Sistemas De Información Geográfica Y Evaluación Multicriterio En La Ordenación Del Territorio. Madrid (España): RA-MA

Ibáñez, J., J. Martínez, S. Schnabel. 2007. “Desertification Due To Overgrazing In A Dynamic Commercial Livestock-Grass-Soil System." Ecological Modelling 205 (3-4): 277-288. doi: 10.1016/j.ecolmodel.2007.02.024

IDEAM, IGAC y CORMAGDALENA. 2008. Mapa de Cobertura de la Tierra Cuenca Magdalena-Cauca: Metodología. CORINE Land Cover adaptada para Colombia a escala 1:100.000. Bogotá (Colombia): Instituto de Hidrología, Meteorología y Estudios Ambientales, Instituto Geográfico Agustín Codazzi y Corporación Autónoma Regional del río Grande de La Magdalena.

Lara, L., L. Rasche, y U. A. Schneider. 2017. “Environmental Modelling Y Software Modeling Land Suitability for Coffea arabica L. In Central America." Environmental Modelling and Software 95:196-209. doi: 10.1016/j.envsoft.2017.06.028

Li, X., X. Hou, Z. Liu, F. Guo, Y. Ding, y J. Duan. 2016. "Long-Term Overgrazing-Induced Changes in Topsoil Water-Retaining Capacity in a Typical Steppe.” Rangeland Ecology \& Management 70 (3): 324-330. doi: 10.1016/j.rama.2016.10.002

López, L. B., y J. C. C. García. 2015. “Nitrógeno Edáfico Y Nodulación De Leucaena leucocephala (Lam.) de Wit En Sistemas Silvopastoriles. Acta Agronomica 64 (4): 349-354. doi: 10.15446/acag.v64n3.43488

Malczewski, J. 1999. GIS and multicriteria decision analysis. Nueva York (EEUU): John Wiley and Sons.
Martínez-Valderrama, J., J. Ibáñez, G. Del Barrio, F. J. Alcalá, M. E. Sanjuán, A. Ruiz, J. Puigdefábregas. 2018. "Doomed To Collapse: Why Algerian Steppe Rangelands Are Overgrazed And Some Lessons To Help Land-Use Transitions." Science of the Total Environment $613-614$ : $1489-1497$. do i : 10.1016/j.scitotenv.2017.07.058

Mazo, N., J. Rubiano, y A. Castro. 2016. "Sistemas Agroforestales Como Estrategia Para El Manejo De Ecosistemas De Bosque Seco Tropical En El Suroccidente Colombiano Utilizando Los SIG”. Revista Colombiana de Geografia 25 (1): 65-77.

Mora A. M., y R. T. Ramírez. 2013. Propuesta De Zonificación Ambiental Para Las Microcuencas De Los Ríos Blanco Y Cuipilapa, Bagaces Costa Rica. Tesis de Grado. Universidad de Costa Rica. San José (Costa Rica)

Obade, V., y R. Lal. 2013. “Assessing Land Cover And Soil Quality By Remote Sensing And Geographical Information Systems (GIS).” CATENA 104: 77-92.

Palma-López D. J., J. Cisneros, E. Moreno, J. A. Rincón-Ramírez. 2007. Suelos De Tabasco: Su Uso Y Manejo Sustentable. Tabasco (México): Colegio de Postgraduados.

Parrado Grajales, F. J. 2004. Evaluación De La Aptitud De Tierras Para Los Cultivos De Repollo, Maíz Y Zanahoria Bajo Dos Diferentes Tecnologías En La Microcuenca La Soledad, Valle De Ángeles, Honduras. Tesis de Maestría. CATIE. Turrialba (Costa Rica).

Saaty, T. L. 2008. "Decision Making With The Analytic Hierarchy Process." International Journal of Services Sciences 1 (1): 83-98. doi:10.1504/IJSSCI.2008.017590

Sakadevan M., y M. Nguyen. 2017. "Livestock Production and Its Impact on Nutrient Pollution and Greenhouse Gas Emissions." Advances in Agronomy 141: 147-184. 
Sánchez, S.; M. Hernández, y F. Ruz. 2011. “Alternativas De Manejo De La Fertilidad Del Suelo En Ecosistemas Agropecuarios." Pastos y Forrajes 34 (4): 375-392.

Sharma, R. P., R. S. Singh, S. K. Singh, P. S. Naik, y B. Singh. 2014. "Health Of Soil Supporting Vegetable Cultivation In Peri-Urban Areas." International Journal of Vegetable Science 22 (1): 35-47. doi: 10.1080/19315260.2014. 923549 .

Silberman, J. E., A. L. Anriquez, J. A. Domínguez, C. G. Kunst, y A. S. Albanesi. 2015. "La Cobertura Arbórea Es Un Sistema Silvopastoril Del Chaco Y Su Contribución Diferencial Al Suelo." Ciencia del Suelo 33 (1): 19-29.

Vargas, H., y D. Ponce de León. 2008. “Evaluación De La Aptitud De Las Tierras Del Municipio San José De Las Lajas Para Las Clases Generales De Uso Agrícola Y Ganadero. I. Aptitud Física." Revista Ciencias Tecnicas Agropecuarias 17 (4): 64-69.

Widiatmaka, W. 2016. "Integrated Use Of GIS, AHP And Remote Sensing In Land Use Planning For Tropical High Altitude Vegetable Crops." Journal of Applied Horticulture 18 (2): 87-99. 